\title{
Valoración nutricional in situ de dietas con harina de maracuyá (Passiflora edulis) en sustitución del maíz (Zea mays)
}

\author{
In situ nutritional assessment of diets with passion fruit meal (Passiflora edulis) \\ as replacement of maize (Zea mays)
}

\author{
Adolfo Sánchez Laíño ${ }^{1,3}$, Emma Torres Navarrete ${ }^{1}$, Ítalo Espinoza Guerra ${ }^{1}$, León \\ Montenegro Vivas ${ }^{1}$, Cecilio Barba Capote ${ }^{2}$, Antón García Martínez ${ }^{2}$
}

\section{Resumen}

Se estudiaron los parámetros de degradación ruminal in situ de la harina de maracuyá (Passiflora edulis) incluida en dietas para rumiantes en sustitución del maíz. Se aplicó un diseño de bloques completos al azar con cuatro tratamientos $(0,10,20$ y $30 \%$ [T1, T2, T3 y T4, respectivamente] de inclusión de harina de maracuyá), tres bloques (bovinos con rumen fistulado) y siete tiempos de incubación ( 0 , 3, 6, 12, 24, 48 y 72 horas). Las diferencias entre medias de tratamientos se establecieron mediante la prueba de Tukey $(p<0.05)$. $\mathrm{T} 1$ y T2 presentaron la mayor $(\mathrm{p}<0.05)$ degradabilidad de la materia seca y de la materia orgánica a las 48 y $72 \mathrm{~h}$ de incubación, respectivamente, mientras que la fibra detergente neutra y la fibra detergente ácida tuvieron una mayor degradabilidad en T4 y T3 a las 48 y 72 h de incubación. Los parámetros de cinética ruminal no fueron significativos en ninguna de las variables evaluadas. Los resultados demuestran que la harina de maracuyá puede ser utilizada en sustitución del maíz hasta en un $10 \%$ en dietas para rumiantes en el Litoral ecuatoriano.

Palabras clave: degradabilidad ruminal; residuos agroindustriales; alimentación de rumiantes; cinética ruminal

\footnotetext{
${ }^{1}$ Carrera de Ingeniería Zootécnica, Facultad de Ciencias Pecuarias, Universidad Técnica Estatal de Quevedo, Mocache, Los Ríos, Ecuador

2 Departamento de Producción Animal, Universidad de Córdoba, España

${ }^{3}$ E-mail: arsanchez@uteq.edu.ec
}

Financiamiento del estudio a través de Fondo Competitivo de Investigación Ciencia y Tecnología (FOCICYT) de la Universidad Técnica Estatal de Quevedo en el proyecto de Investigación «Composición química, ensilabilidad, estabilidad aeróbica de dos subproductos agrícolas para la alimentación de rumiantes en el Litoral ecuatoriano»

Recibido: 22 de marzo de 2018

Aceptado para publicación: 27 de octubre de 2018 
The parameters of in situ ruminal degradation of passion fruit meal (Passiflora edulis) included in diets for ruminants as replacement of maize were studied. A randomized complete block design was applied with four treatments $(0,10,20$ and 30\% [T, T2, T3 and T4, respectively] of inclusion of passion fruit meal), three blocks (rumen-fistulated bovine) and seven incubation times ( $0,3,6,12,24,48$ and 72 hours). The differences between means were established using the Tukey test $(\mathrm{p}<0.05)$. T1 and T2 showed the highest $(\mathrm{p}<0.05)$ degradability of dry matter and organic matter at 48 and $72 \mathrm{~h}$ of incubation, respectively, while neutral detergent fibre and acid detergent fibre had a greater degradability in T4 and T3 at 48 and $72 \mathrm{~h}$ of incubation. The ruminal kinetics parameters were not significant in any of the variables evaluated. The results show that passion fruit meal can be used to replace maize up to $10 \%$ in diets for ruminants in the Ecuadorian littoral.

Key words: ruminal degradability; agro-industrial waste; ruminant feeding; ruminal kinetic

\section{INTRODUCCIÓN}

Las mayores precipitaciones en el Ecuador se concentran en los meses de diciembre a mayo, siendo la época de mayor producción forrajera (Espinoza-Guerra et al., 2016). En las regiones tropicales, las estrategias de alimentación de los rumiantes se basan en gran medida en el aprovechamiento directo de pastos, así como en la utilización de los recursos disponibles localmente, tales como los residuos agroindustriales (Bocquier y González-García, 2010), cuya producción y valor nutritivo descienden drásticamente en la época seca (junio a diciembre). En este periodo, el ganado no logra cubrir sus requerimientos nutritivos, constituyéndose en la principal limitante para mantener una producción estable durante el año (Nieto y Vimos, 1998; Guevara et al., 2009).

Se dispone de una gran diversidad de residuos agroindustriales que pueden o podrían ser utilizados en la alimentación de rumiantes, existiendo una coyuntura económica y social internacional favorable a su reciclaje como alimentos para los animales de granja (Manterola et al., 2000; Martín,
2009). Muchos de estos restos se pierden o son subutilizados, debido al conocimiento limitado sobre su valor nutritivo y de sus limitaciones en la respuesta del animal al incluirlos en la dieta.

La producción mundial de maracuyá (Passiflora edulis) es de importancia en países tropicales y subtropicales (Nhut et al., 2007), destacando Brasil con 450000 t, Ecuador con $85000 \mathrm{t}$ y Colombia con $75000 \mathrm{t}$ (SICA, 2009). La extracción de la pulpa para la obtención de zumo genera una mezcla de pericarpios y semillas como residuo, que representan el 53 y $21 \%$ del peso del fruto, respectivamente (Oliveira et al., 2002), a lo que se debe sumar el costo que las industrias deben asumir por concepto de disposición final apropiada del residuo, en cumplimiento de las medidas medioambientales establecidas.

Estos subproductos son de escaso valor biológico, debido al reducido contenido proteico (2-6\%), digestibilidad (25-40\%), extracto libre de nitrógeno (34\%) y un bajo perfil mineral (7.8\%) (Carnevali et al., 1991; Pedraza et al., 1994), por lo que su uso en alimentación se orienta fundamentalmente a rumiantes (Vieira et al., 1999). Es por ello 
que se requiere establecer pautas de utilización dentro de las dietas (Castillo y Onetti, 2002), y para lo cual es necesario determinar su composición química y establecer su valor nutritivo (Preston, 1986). En el caso de los rumiantes, el valor nutritivo y la inclusión de los alimentos en las raciones dependen de la cinética de degradación ruminal de los componentes. La sustitución parcial del maíz por cascara de maracuyá se justifica por el contenido de energía, el alto contenido de fibra que puede permitir mejorar las funciones del rumen e incluso reducir las posibilidades de timpanismo de los rumiantes; además, es de muy bajo costo. Por tanto, el objetivo del presente trabajo fue determinar la valoración nutricional in situ de dietas con harina de maracuyá en sustitución del maíz.

\section{Materiales y Métodos}

La investigación se llevó a cabo en el Laboratorio de Rumiología y Metabolismo Nutricional (RUMEN) de la Universidad Técnica Estatal de Quevedo (UTEQ), provincia de Los Ríos, Ecuador. La cáscara de maracuyá $(300 \mathrm{~kg})$ se obtuvo en una sola oportunidad de la empresa Tropifrutas, localizada en el cantón Quevedo. El residuo fresco fue trasladado a las instalaciones de la UTEQ y secado al sol. El análisis proximal del residuo de maracuyá se presenta en el Cuadro 1.

Las muestras de cada tratamiento fueron procesadas en un molino de laboratorio (Thomas Model 4 Wiley ${ }^{\circledR}$ Mill) con criba de $2 \mathrm{~mm}$. El análisis de la materia seca (MS), materia orgánica (MO) y cenizas se realizó mediante los procedimientos establecidos por la AOAC (2013) y el de la fibra detergente neutra $(F D N)$ y fibra detergente ácida (FDA), según Van Soest (1994) (Cuadro 2). Para los análisis de degradación ruminal se utilizaron bolsas de nylon Ankom F-57 (Ankon Technology, EEUU) de 10x19 cm y $50 \mu \mathrm{m}$
Cuadro 1. Composición química en base a materia seca (MS) del residuo de maracuyá (Passiflora edulis Sims)

\begin{tabular}{lc}
\hline Componentes & Porcentaje \\
\hline Materia seca (MS) & 15.10 \\
Materia orgánica (MO) & 88.27 \\
Cenizas & 11.73 \\
Proteína bruta (PB) & 8.77 \\
Fibra detergente neutra (FDN) & 61.54 \\
Fibra detergente ácida (FDA) & 36.39 \\
\hline
\end{tabular}

Fuente: Laboratorio de Rumiología y Metabolismo Nutricional (RUMEN), UTEQ-FCP. 2017

de poro. Las bolsas fueron fabricadas en poliéster/polietileno con filamentos extruidos en una matriz de tres dimensiones (Giraldo et al., 2007). En cada bolsa se colocaron $10 \mathrm{~g}$ de muestra molida y luego se sellaron con prensa térmica (Heat Sealer 1915, Ankon Technology). Se incubaron seis bolsas por tratamiento y tiempo de incubación; además, se incluyeron dos bolsas vacías en cada tiempo de incubación que sirvieron como blancos para determinar el factor de corrección para el efecto del lavado.

Se aplicó un diseño de bloques completos al azar (DBCA) con cuatro niveles de inclusión (tratamientos) de harina de maracuyá en sustitución del maíz en la dieta: T1, control (sin harina de maracuyá) y T2, T3 y T4 con 10 , 20 y $30 \%$ de harina de maracuyá. Se evaluó la cinética de degradación ruminal in situ de la MS, MO, FDN y FDA en siete tiempos de incubación (0, 3, 6, 12, 24, 48 y 72 horas). Se utilizaron tres bovinos Brahman castrados y fistulados en el rumen, con pesos de $450 \pm 20$ kg. Cada animal representó un bloque. Para la diferencia entre medias se aplicó la prueba de Tukey $(p<0.05)$, utilizando el procedimiento de los modelos lineales generales (GLM) del SAS v. 9.1. 
Cuadro 2. Composición del concentrado (en porcentaje de base seca)

\begin{tabular}{lcccc}
\hline & \multicolumn{5}{c}{ Dietas } \\
\cline { 2 - 5 } & D1 & D2 & D3 & D4 \\
\hline Maíz & 57 & 47 & 37 & 27 \\
Harina de maracuyá & - & 10 & 20 & 30 \\
Polvillo de cono & 20 & 20 & 20 & 20 \\
Torta de soya & 18.4 & 18.4 & 18.4 & 18.4 \\
Carbonato de calcio & 1.7 & 1.7 & 1.7 & 1.7 \\
Fosfato monocálcico & 1.4 & 1.4 & 1.4 & 1.4 \\
Sal & 1.5 & 1.5 & 1.5 & 1.5 \\
\hline Composición química (\% & $\mathrm{T} 1$ & $\mathrm{~T} 2$ & $\mathrm{~T} 3$ & \multirow{2}{*}{ T4 } \\
base seca) & 89.98 & 89.96 & 90.35 & 89.45 \\
\hline Materia seca & 90.89 & 90.41 & 89.84 & 89.40 \\
Materia orgánica & 46.68 & 42.03 & 38.20 & 38.56 \\
Fibra detergente neutra & 8.50 & 11.96 & 15.32 & 21.31 \\
Fibra detergente ácida & & & &
\end{tabular}

T1, T2, T3 y T4: 0, 10, 20 y 30\% de harina de maracuyá

Los toros fistulados fueron alimentados con forraje picado de pasto saboya (Panicum maximun J). Las bolsas fueron retiradas del ambiente ruminal al término de los periodos de incubación y se colocaron en una cubeta con agua fría para detener la actividad microbiana. Se lavaron con agua corriente hasta obtener un efluente trasparente. Luego fueron desecadas a temperatura ambiente y de allí en estufa de aire forzado (Memmert UN55, Alemania) por $48 \mathrm{~h} \mathrm{a} 65^{\circ} \mathrm{C}$.

Los parámetros de degradación ruminal de la MS, MO, FDN y FDA se estimaron con el modelo de Ørskov y McDonald (1979) mediante la fórmula $\mathrm{d}=\mathrm{a}+\mathrm{b}\left(1-\mathrm{e}^{-\mathrm{kdt}}\right)$, donde $d$ $=$ Degradación de la MS, MO, FDN y FDA, $a=$ fracción soluble o el sustrato rápidamente degradable, $b=$ fracción potencialmente degradable, no soluble, $a+b=$ degradabilidad potencial $(\%), \mathrm{kd}=$ tasa de degradación de la fracción $\mathrm{b}\left(\% \mathrm{~h}^{-1}\right)$ y $c=$ fracción no degradable.

\section{Resultados y Discusión}

La degradabilidad in situ de la materia seca (DISMS) de las dietas experimentales no presentaron diferencias a las $0,3,6$ y $12 \mathrm{~h}$ de incubación, pero T3 fue diferente a T1 y $\mathrm{T} 2$ a las $24 \mathrm{~h}(\mathrm{p}<0.05), \mathrm{T} 2$, T 3 y T4 fueron diferentes a T0 a las $48 \mathrm{~h}$, siendo la menor degradabilidad en T4 ( $<<0.05)$, y T2, T 3 y $\mathrm{T} 4$ fueron diferentes a T0 a las $72 \mathrm{~h}$, siendo las menores degradabilidades en T3 y T4 $(\mathrm{p}<0.05)$ (Cuadro 3). La degradabilidad de la MS de T1, T2 y T3 a las 48 y $72 \mathrm{~h}$ de incubación superan a las reportadas por Villela et al. (1997), quienes reportan valores de $63.65,62.38$ y $63.46 \%$, con una tasa de pasaje de $4.8 \% / \mathrm{h}$, al evaluar la cinética de degradación ruminal de residuos de tres variedades de maracuyá (amarilla, roja, dulce). De igual manera superan los resultados de Pimentel et al. (2005). Los valores de degradabilidad de la MS observados en fun- 
Cuadro 3. Degradabilidad y parámetros de la cinética ruminal de la materia seca (MS) en dietas con harina de maracuyá (Phasiflora edullis) en siete periodos de incubación in situ

\begin{tabular}{cccccc}
\hline \multirow{2}{*}{ Incubación (h) } & \multicolumn{4}{c}{ Harina de maracuyá (\%) } & \multirow{2}{*}{ EEM $^{1}$} \\
\cline { 2 - 4 } & 0 & 10 & 20 & 30 & \\
\hline 0 & $12.62^{\mathrm{a}}$ & $13.22^{\mathrm{a}}$ & $13.81^{\mathrm{a}}$ & $13.43^{\mathrm{a}}$ & 0.247 \\
3 & $17.43^{\mathrm{a}}$ & $16.47^{\mathrm{a}}$ & $17.55^{\mathrm{a}}$ & $16.40^{\mathrm{a}}$ & 0.413 \\
6 & $23.92 \mathrm{a}$ & $23.44^{\mathrm{a}}$ & $22.99^{\mathrm{a}}$ & $22.64^{\mathrm{a}}$ & 0.775 \\
12 & $41.02^{\mathrm{a}}$ & $42.21^{\mathrm{a}}$ & $40.99^{\mathrm{a}}$ & $40.78^{\mathrm{a}}$ & 0.741 \\
24 & $60.89^{\mathrm{a}}$ & $60.75^{\mathrm{a}}$ & $59.67^{\mathrm{ab}}$ & $57.91^{\mathrm{b}}$ & 0.429 \\
48 & $67.68^{\mathrm{a}}$ & $65.40^{\mathrm{b}}$ & $64.45^{\mathrm{b}}$ & $62.19^{\mathrm{c}}$ & 0.630 \\
72 & $69.10^{\mathrm{a}}$ & $66.71^{\mathrm{b}}$ & $64.91^{\mathrm{c}}$ & $63.25^{\mathrm{c}}$ & 0.673 \\
Fracciones & & & & & \\
$a$ & $9.17^{\mathrm{a}}$ & $9.30^{\mathrm{a}}$ & $9.55^{\mathrm{a}}$ & $9.04^{\mathrm{a}}$ & 0.221 \\
$b$ & $59.91^{\mathrm{a}}$ & $59.68^{\mathrm{a}}$ & $58.41^{\mathrm{a}}$ & $57.84^{\mathrm{a}}$ & 0.778 \\
$k d$ & $6.34^{\mathrm{a}}$ & $6.20^{\mathrm{a}}$ & $6.13^{\mathrm{a}}$ & $6.70^{\mathrm{a}}$ & 0.696 \\
$c$ & $30.92^{\mathrm{a}}$ & $31.02^{\mathrm{a}}$ & $32.03^{\mathrm{a}}$ & $33.12^{\mathrm{a}}$ & 0.118 \\
\hline
\end{tabular}

$a, b, c$ Promedios con letras distintas difieren estadísticamente $(p<0.05)$

${ }^{1}$ Error estándar de la media

$a=$ fracción soluble o el sustrato rápidamente degradable, $b=$ fracción potencialmente degradable, no soluble, $\mathrm{kd}$ = tasa de degradación, $c$ = fracción no degradable

Cuadro 4. Degradabilidad y parámetros de la cinética ruminal de la materia orgánica (MO) en dietas con harina de maracuyá (Phasiflora edullis) en siete periodos de incubación

\begin{tabular}{cccccc}
\hline \multirow{2}{*}{ Incubación (h) } & \multicolumn{4}{c}{ Harina de maracuyá (\%) } & \multirow{2}{*}{ EEM $^{1}$} \\
\cline { 2 - 4 } & 0 & 10 & 20 & 30 & \\
\hline 0 & $9.62^{\mathrm{a}}$ & $8.41^{\mathrm{a}}$ & $9.25^{\mathrm{a}}$ & $8.10^{\mathrm{a}}$ & 0.358 \\
3 & $13.15^{\mathrm{a}}$ & $13.32^{\mathrm{a}}$ & $13.57^{\mathrm{a}}$ & $11.90^{\mathrm{a}}$ & 0.476 \\
6 & $20.22^{\mathrm{a}}$ & $19.66^{\mathrm{ab}}$ & $18.75^{\mathrm{b}}$ & $18.56^{\mathrm{b}}$ & 0.823 \\
12 & $40.46^{\mathrm{a}}$ & $41.75^{\mathrm{a}}$ & $39.47^{\mathrm{a}}$ & $39.63^{\mathrm{a}}$ & 0.654 \\
24 & $61.51^{\mathrm{a}}$ & $61.03^{\mathrm{a}}$ & $59.72^{\mathrm{ab}}$ & $57.53^{\mathrm{b}}$ & 0.746 \\
48 & $71.79^{\mathrm{a}}$ & $69.28^{\mathrm{ab}}$ & $65.94^{\mathrm{bc}}$ & $64.31^{\mathrm{c}}$ & 0.999 \\
72 & $74.14^{\mathrm{a}}$ & $70.07^{\mathrm{b}}$ & $67.54^{\mathrm{bc}}$ & $65.16^{\mathrm{c}}$ & 1.032 \\
Fracciones & & & & & \\
$a$ & $4.24^{\mathrm{a}}$ & $5.38^{\mathrm{a}}$ & $4.35^{\mathrm{a}}$ & $3.86^{\mathrm{a}}$ & 0.303 \\
$b$ & $69.77^{\mathrm{a}}$ & $67.01^{\mathrm{a}}$ & $67.24^{\mathrm{a}}$ & $65.59^{\mathrm{a}}$ & 1.036 \\
$k d$ & $5.90^{\mathrm{a}}$ & $5.87^{\mathrm{a}}$ & $5.84^{\mathrm{a}}$ & $6.43^{\mathrm{a}}$ & 1.123 \\
$c$ & $25.98^{\mathrm{a}}$ & $27.06^{\mathrm{a}}$ & $28.41^{\mathrm{a}}$ & $30.55^{\mathrm{a}}$ & 0.143 \\
\hline
\end{tabular}

$a, b, c$ Promedios con letras distintas difieren estadísticamente $(p<0.05)$

1 Error estándar de la media

$a=$ fracción soluble o el sustrato rápidamente degradable, $b=$ fracción potencialmente degradable, no soluble, $k d$ = tasa de degradación, $c$ = fracción no degradable 
Cuadro 5. Degradabilidad y parámetros de la cinética ruminal de la fibra detergente neutra (FDN) en dietas con harina de maracuyá (Phasiflora edullis) en siete periodos de incubación

\begin{tabular}{cccccc}
\hline \multirow{2}{*}{ Incubación (h) } & \multicolumn{4}{c}{ Harina de maracuyá (\%) } & \multirow{2}{*}{ EEM $^{1}$} \\
\cline { 2 - 5 } & 0 & 10 & 20 & 30 & \\
\hline 0 & $28.90^{\mathrm{c}}$ & $31.93^{\mathrm{bc}}$ & $36.15^{\mathrm{ab}}$ & $38.86^{\mathrm{a}}$ & 1.236 \\
3 & $29.70^{\mathrm{d}}$ & $34.21^{\mathrm{c}}$ & $39.42^{\mathrm{b}}$ & $43.89^{\mathrm{a}}$ & 1.638 \\
6 & $32.99^{\mathrm{c}}$ & $34.87^{\mathrm{c}}$ & $41.46 \mathrm{~b}$ & $46.44^{\mathrm{a}}$ & 1.623 \\
12 & $34.51^{\mathrm{b}}$ & $37.72^{\mathrm{b}}$ & $45.03^{\mathrm{a}}$ & $50.30^{\mathrm{a}}$ & 1.951 \\
24 & $40.12^{\mathrm{d}}$ & $54.69^{\mathrm{c}}$ & $63.65^{\mathrm{b}}$ & $72.24^{\mathrm{a}}$ & 3.658 \\
48 & $59.63^{\mathrm{d}}$ & $74.18^{\mathrm{c}}$ & $80.44^{\mathrm{b}}$ & $86.46^{\mathrm{a}}$ & 3.029 \\
72 & $65.32^{\mathrm{c}}$ & $78.48^{\mathrm{b}}$ & $81.02^{\mathrm{b}}$ & $88.33^{\mathrm{a}}$ & 2.573 \\
Fracciones & & & & & \\
$a$ & $34.57^{\mathrm{a}}$ & $35.41^{\mathrm{a}}$ & $37.18^{\mathrm{a}}$ & $37.35^{\mathrm{a}}$ & 1.377 \\
$b$ & $42.08^{\mathrm{a}}$ & $44.44^{\mathrm{a}}$ & $43.53^{\mathrm{a}}$ & $45.16^{\mathrm{a}}$ & 1.176 \\
$k d$ & $7.11^{\mathrm{a}}$ & $7.96^{\mathrm{a}}$ & $7.28^{\mathrm{a}}$ & $9.17^{\mathrm{a}}$ & 2.175 \\
$c$ & $23.35^{\mathrm{a}}$ & $20.15^{\mathrm{a}}$ & $19.30^{\mathrm{a}}$ & $17.49^{\mathrm{a}}$ & 0.62 \\
\hline
\end{tabular}

Cuadro 6. Degradabilidad y parámetros de la cinética ruminal de la fibra detergente ácida (FDA) en dietas con harina de maracuyá (Phasiflora edullis) en siete periodos de incubación

\begin{tabular}{cccccc}
\hline \multirow{2}{*}{ Incubación (h) } & \multicolumn{4}{c}{ Harina de maracuyá (\%) } & \multirow{2}{*}{ EEM $^{1}$} \\
\cline { 2 - 5 } & 0 & 10 & 20 & 30 & \\
\hline 0 & $7.50^{\mathrm{c}}$ & $11.96^{\mathrm{b}}$ & $17.67^{\mathrm{b}}$ & $23.03^{\mathrm{a}}$ & 1.780 \\
3 & $7.76^{\mathrm{d}}$ & $13.11^{\mathrm{c}}$ & $19.31^{\mathrm{b}}$ & $24.20^{\mathrm{a}}$ & 1.876 \\
6 & $7.78^{\mathrm{d}}$ & $13.02^{\mathrm{c}}$ & $19.93^{\mathrm{b}}$ & $24.37^{\mathrm{a}}$ & 1.926 \\
12 & $9.87^{\mathrm{c}}$ & $14.60^{\mathrm{b}}$ & $24.80^{\mathrm{a}}$ & $26.24^{\mathrm{a}}$ & 2.082 \\
24 & $16.89^{\mathrm{d}}$ & $27.24^{\mathrm{c}}$ & $34.11^{\mathrm{b}}$ & $42.27^{\mathrm{a}}$ & 2.854 \\
48 & $29.85^{\mathrm{d}}$ & $40.91^{\mathrm{c}}$ & $47.86^{\mathrm{b}}$ & $53.94^{\mathrm{a}}$ & 2.721 \\
72 & $31.31^{\mathrm{d}}$ & $42.71^{\mathrm{c}}$ & $49.36^{\mathrm{b}}$ & $56.21^{\mathrm{a}}$ & 2.780 \\
Fracciones & & & & & \\
$a$ & $10.86^{\mathrm{a}}$ & $11.51^{\mathrm{a}}$ & $13.73^{\mathrm{a}}$ & $15.41^{\mathrm{a}}$ & 1.726 \\
$b$ & $48.59^{\mathrm{a}}$ & $47.01^{\mathrm{a}}$ & $45.41^{\mathrm{a}}$ & $46.87^{\mathrm{a}}$ & 1.425 \\
$k d$ & $1.78^{\mathrm{a}}$ & $1.87^{\mathrm{a}}$ & $1.79^{\mathrm{a}}$ & $2.09^{\mathrm{a}}$ & 2.212 \\
$c$ & $40.55^{\mathrm{a}}$ & $41.48^{\mathrm{a}}$ & $40.86^{\mathrm{a}}$ & $37.72^{\mathrm{a}}$ & 0.151 \\
\hline
\end{tabular}


ción del tiempo de incubación demuestran un alto grado de ajuste $\left(\mathrm{R}^{2}=0.9937,0.9929\right.$, 0.9913 y 0.9924$)$.

La degradabilidad ruminal in situ de la materia orgánica (DISMO) de las dietas durante los periodos de incubación de 0,3 y 12 $\mathrm{h}$ no se vio afectado por la inclusión de la harina de maracuyá; sin embargo, en mayores tiempos de incubación se encontró que la inclusión de harina de maracuyá provocó una disminución de la degradabilidad $(\mathrm{p}<0.05$; Cuadro 4). La degradabilidad de la MS y MO de T1, T2, T3 y T4 a las 48 y $72 \mathrm{~h}$ de incubación superan los valores de 60.0 y $58.2 \%$, respectivamente, reportados por Lousada et al. (2005), al evaluar el consumo $\mathrm{y}$ digestibilidad de subproductos del procesamiento de frutas en ovinos. Los valores de degradabilidad de la MO observados en función del tiempo de incubación demuestran un alto grado de ajuste $\left(\mathrm{R}^{2}=0.9966,0.9914\right.$, 0.9877 y 0.9897$)$.

La mayor respuesta en DISMS y DISMO puede deberse a la presencia de carbohidratos solubles aportados por el maíz en las dietas del presente estudio y, por tanto, menor cantidad de paredes celulares, que limiten la degradabilidad a nivel ruminal, posibilitando un ambiente con mejor perfil de nutrientes para los microorganismos degradadores de estos sustratos. Noguera et al. (2006) aseguran que el metabolismo de los microorganismos ruminales se regula por la cantidad de carbohidratos no estructurales (CNE) en la ración y el tipo de procesamiento al cual hayan sido sometidos (químicos o físicos), ya que al aumentar la densidad energética con CNE procesados se promueve un rápido crecimiento microbiano y la producción de ácidos grasos volátiles (AGV).

T4 presentó la mejor repuesta para la degradabilidad in situ de la FDN (DISFDN) y FDA (DISFDA) $(p<0.05)$ en cada periodo de incubación, excepto para la DISFDN a las $0 \mathrm{~h}$ y para la DISFDA a las $12 \mathrm{~h}$ de incubación donde T3 y T4 presentaron resultados similares (Cuadros 5 y 6). Se puede observar que al incrementar los porcentajes de inclusión de harina de maracuyá en la dieta y en los periodos de incubación, se aumenta la DISFDN y la DISFDA. Las degradabilidades de la FDN de los cuatro tratamientos a las 48 y $72 \mathrm{~h}$ de incubación superan los valores de $43.25,40.00$ y $34.61 \%$ reportados por Villela et al. (1997). En forma similar, varios de los valores de degradabilidad de la FDN a las 24, 48 y $72 \mathrm{~h}$ de incubación superan el $56.2 \%$ encontrado por Lousada et al. (2005), quienes evaluaron el consumo y digestibilidad de subproductos del procesamiento de frutas para ovinos. Los valores de degradabilidad de la FDN y FDA observados en función del tiempo de incubación demuestran un alto grado de ajuste $\left(\mathrm{R}^{2}=0.9785\right.$, $0.9970,0.9979$ y $0.9938 ; \mathrm{R}^{2}=0.9933,0.9973$, 0.9981 y 0.9939 , respectivamente).

Los parámetros de cinética ruminal no fueron significativos en ninguna de las variables evaluadas (MS, MO, FDN, FDA). Se reconoce que es posible mejorar el consumo de nutrientes en los animales al incrementar la digestibilidad de las fracciones potencialmente digestibles o la velocidad de paso de las fracciones no digeribles en rumen (Barahona y Sánchez, 2005; Correa, 2002; Forbes, 2005), pero estas estrategias deben desarrollarse con un adecuado conocimiento de la cinética de los forrajes en su tránsito por el tracto digestivo de los animales (Naranjo y Cuartas, 2011).

\section{Conclusión}

El uso de harina de cáscara de maracuyá en dietas para rumiantes representa una alternativa viable en niveles de hasta el $10 \%$ de sustitución del maíz por los similares valores de degradabilidad demostrados con relación a la dieta control en el máximo tiempo de incubación estudiado (72 horas). 


\section{Literatura Citada}

1. [AOAC] Association of Official Agricultural Chemists. 2013. Official methods of analysis. $17^{\text {th }}$ ed. $2^{\text {nd }}$ Revision. USA: AOAC. 600 p.

2. Barahona R, Sánchez S. 2005. Limitaciones físicas y químicas de la digestibilidad de pastos tropicales y estrategias para aumentarla. Rev Corpoica 6: 69-82.

3. Bocquier F, González-García E. 2010. Sustainability of ruminant agriculture in the new context: feeding strategies and features of animal adaptability into the necessary holistic approach. Animal 4: 1258-1273. doi: 10.1017/S1751731110001023

4. Carnevali A, Shultz T, Shultz E, Chico C. 1991. Suplementación del heno de pobre calidad con melaza y urea. Agronomía Trop 2: 565-572.

5. Castillo A, Onetti S. 2002. Origen y composición química de los subproductos agroindustriales. En: Los subproductos agroindustriales en la alimentación de los rumiantes. INTA, Argentina: [Internet]. Disponible en: https://www.agro.uba.ar/ sites/default/files/agronomia/ subproductos_suplementacion.pdf

6. Correa HJ. 2002. Limitaciones metabólicas para la producción bovina en el trópico bajo. En: Estrategias de alimentación en la ganadería y su impacto en la productividad. Colombia: Univ. Nacional de Colombia. p 34.

7. Espinoza-Guerra I, Pérez-Oñate C, Montenegro-Vivas L, Sánchez-Laíño A, García-Martínez A, MartínezMarín A. 2016. Composición química y cinética de degradación ruminal in vitro del ensilado de pasto saboya (Megathyrsus maximus) con niveles crecientes de inclusión de residuo de maracuyá (Passiflora edullis Sims). Rev Cient 26: 402-407.

8. Forbes JM. 2005. Voluntary feed intake and diet selection. In: Dijkstra J, Forbes JM, France J (eds). $2^{\text {nd }}$ ed. Quantitative aspects of ruminant digestion and metabolism. CABI Publishing. p 607-627.

9. Giraldo LA, Gutiérrez LA, Rúa C. 2007. Comparación de dos técnicas in vitro e in situ para estimar la digesti-bilidad verdadera en varios forrajes tropicales. Rev Colomb Cienc Pec 20: 269-279.

10. Guevara E, Villamide MJ, Rodríguez I, Gil JL, Blanco, C. 2009. Minisilos de gramíneas y leguminosas. Una alternativa para la alimentación de pequeños rumiantes. INIA Divulga 14: 2-7.

11. Lousada JE, Miranda J, Rodríguez N, Pimentel J, Braga R. 2005. Consumo e digestibilidade de subprodutos do processamento de frutas em ovinos. Rev Bras Zootecn 34: 659-669. doi: 10.1590/ S1516-35982005000200036

12. Manterola B, Cerda D, Mira J. 2000. Los residuos agrícolas y su uso en la alimentación de rumiantes. Chile: Ed FIA. $222 \mathrm{p}$.

13. Martín P. 2009. El uso de residuales agroindustriales en la alimentación animal en Cuba: pasado, presente y futuro. Avances Invest Agropec 13: 3-10

14. Naranjo JF, Cuartas CA. 2011. Caracterización nutricional y de la cinética de degradación ruminal de algunos de los recursos forrajeros con potencial para la suplementación de rumiantes en el trópico alto de Colombia. CES Med Vet Zootec 6: 9-19.

15. Nhut DT, Khiet B, Thi N, Thuy D, Duy N, Hai N, Huyen P. 2007. High frequency shoot formation of yellow passion fruit (Passiflora edulis $f$. flavicarpa) via thin cell layer (TCL) technology. In: Jain SM, Häggman $\mathrm{H}$ (eds). Protocols for micropropagation of woody trees and fruits. The Netherlands: Springer. p 417-426.

16. Nieto C, Vimos M. 1998. Producción y procesamiento de quinua en Ecuador. Proyecto de Cooperación Técnica 3P90-0160. Instituto Nacional Autónomo de Investigaciones Agropecuarias. [Internet]. Disponible en: https://idl-bncidrc.dspacedirect.org/bitstream/handle/ 10625/13343/100162.pdf? sequence $=1$ 
17. Noguera RR, Ramírez IC, Bolívar DM. 2006. Efecto de la inclusión de papa (Solanum tuberosum) en la cinética de fermentación in vitro del pasto kikuyo (Pennisetum clandestinum). Livestock Res Rural Develop 18(5). [Internet]. Disponible en: http://www.lrrd.org/lrrd18/ 5/nogu18062.htm

18. Oliveira LF, Nascimento M, Borges M, Ruback S. 2002. Aproveitamento alternativo da casca do maracujá amarelo (Passiflora edulis F. flavicarpa deg) para producao de doce em calda. Food Sci Tech-Brazil 22: 259-262. doi: 10.1590/ S0101-20612002000300011

19. Orskov ER, McDonald I. 1979. The estimation of protein degradability in the rumen form incubation measurements weighted according to rate of passage. J Agr Sci 92: 499-503. doi: 10.1017/ S0021859600063048

20. Pedraza R, Vita M, González M. 1994. Composición química y degradabilidad ruminal de suplementos elaborados con alta integración de subproductos agroindustriales. Rev Prod Anim 8: 32-37.

21. Pimentel P, De Moraes SA, Guimarães A, Silva M, Benevides Y, Neiva J, De Aquino D. 2005. Degradabilida de in situ da matéria seca de subprodutos do abacaxi, castanha de caju, coco e maracujá. XLII Reuniao Anual da Sociedade Brasielira de Zootecnia. Goiania, Goias.
22. Preston T. 1986. Better utilization of crop residues and by-products in animal feeding: research guidelines. 2. A practical manual for research workers. FAO. [Internet]. Available in: http:// www.fao.org/docrep/003/x6554e/ $\mathrm{x} 6554 \mathrm{e} 00 . \mathrm{htm}$.

23. SICA [Servicio de Información Agropecuaria, Ministerio de Agricultura y Ganadería]. 2009. Boletín situacional. Maracuyá. [Internet]. Disponible en: http://sipa.agricultura.gob.ec/ index.php/maracuya/boletinessituacionales-maracuya-ecuador

24. Van Soest P. 1994. Nutritional ecology of the ruminant. $2^{\text {nd }}$ ed. Ithaca, NY: Cornell University Press. $476 \mathrm{p}$.

25. Vieira C, Vasquez, H, Silva J. 1999. Composição químico-bromatológica e degradabilidade in situ da matéria seca, proteína bruta e fibra em detergente neutro da casca do fruto de três variedades de maracujá (Passiflora spp). Rev Bras Zootecn 28: 1148-1158. doi: 10.1590/ S1516-35981999000500034.

26. Villela C, Maldonado H, Coelho JF, Fernández AM. 1997. Parâmetros da degradação e cinética ruminal da digestão de resíduos (casca) de três variedades de maracujá (Passiflora spp.). Arch Latinoam Prod Anim 5(Suppl 1): 202-204. 